\title{
Effects of response requirements on the location of clicks superimposed on sentences*
}

\author{
MICHAEL R. SEITZ \\ School of Human Communication Disorders \\ .McGill Liniversitr, Montreal, Quebec. Canada \\ and \\ BRUCE A. WEBER \\ iniversity of Wasington. Seattle. Washington 98195
}

\begin{abstract}
Two methods of identifying the location of clicks superimposed on sentences were compared. When Ss first wrote out the entire sentence and then marked the location of the clicks, the perceived clicks tended to migrate toward the major constituent breaks of the sentences. This trend was not observed when Ss responded by marking the position of clicks on prepared scripts of the stimulus sentence. In addition, both response procedures resulted in a significant trend for the perceived clicks to be located before the actual click locations. The findings of this study resolved the conflicting results obtained from previous investigations in this area.
\end{abstract}

There has been a debate concerning the postulated correlation between acoustic output and phonemes of English. Ladefoged and Broadbent (1960) felt that there was not a one-to-one correlation between the acoustic output and the phoneme. To test this hypothesis. they recorded simple sentences or strings of digits on one channel of a stereo recording. with an extraneous sound (either a click or a narrow band of white noise) recorded on the other channel in such a manner that it coincided with a specific portion of the speech stimuli on the first channel. The Ss listened to the speech stimuli presented to one ear and the extraneous sound presented to the other ear. The Ss were then asked to indicate where they felt the "superimposed" sound occurred on the speech stimuli by noting the word or words. The Ss accomplished this task either by writing out the word where the slick occurred or by marking a siript of the speech stimuli which coincided with the occurrence of the extraneous sound. Judgments of extraneous sound location erred by an average of two words from the objective placement of the sound on the stimulus material. with most of the errors indicating that the extraneous sound was perceived as occurring before the objective location. The authors explained the tendency to mark the extraneous sound prior to its actual cocurrence oy citing Titchener's law of prior entry (Titchener. 1909). In brief. Titchener's law say's if an $\mathrm{S}$ is expecting a certain stimulus. such as a click. along with some other unknown stimuli. the $S$ will perceive the expected stimulus faster than the unexpected stimuli. Thus. while Ladefoged and Broadbent did not speculate on the size of the perceptual units. they did interpret errors in location of the extraneous noise as supporting their contention that speech perception occurred in

* This study was part of a PhD dissertation by the first author under the direction of the second author. The authors wish to thank Donald Doehring for his suggestions for the final scrision of this paper units larger than a single phoneme.

Fodor and Bever (1965), citing the Ladefoged and Broadbent data, speculated that the listener, using the intuitive knowledge he possessed about the structure of his native language (Chomsky, 1965), would analyze any incoming linguistic material into units that corresponded to the underlving relationships of actor, action, and object. To accomplish this task, Fodor and Bever suggested that the initial perceptual analysis occurred in units that corresponded to these sentence constituents. Using a modification of the Ladefoged and Broadbent paradigm. Fodor and Bever hypothesized that clicks superimposed around the major constituent breaks (i.e.. the break that occurs between the end of one constituent or clause and the beginning of the next) would be shifted toward the major constituent break because the perceptual unity of the constituents would resist interruption from any extraneous noises. In contrast to the findings of Ladefoged and Broadbent, Fodor and Bever found only a nonsignificant tendency for the click responses to be placed prior to the objective click locations on the stimulus sentences they used. Rather. the perceived click tended to be moved toward or into the major constituent breaks.

Analysis of the paradigms used by Ladefoged and Broadbent and by Fodor and Bever revealed a difference in methods of identifying the location of the superimposed sounds. Ladefoged and Broadbent required their Ss to mark the location of the perceived sound on typed copies of the stimulus sentences or to write out only the word on which the sound was perceived as occurring. In contrast. Fodor and Bever required their Ss to write out the stimulus sentence and then to indicate the location of the superimposed click on the handwritten copy. In subsequent research. those studies which required Ss to locate the superimposed sound on prepared scripts supported the interpretation of Ladefoged and Broadbent (Reber \& Anderson. 1970; 
Scholes. Teas, Nielsen, \& Idzilowski, 1969; Sedge, 1968). while data from studies which required Ss to write out the sentence before locating the superimposed sound supported the interpretation of Fodor and Bever (Abrams \& Bever. 1969; Bever, Kirk, \& Lackner, 1969; Bever. Lackner, \& Kirk, 1969). It appears, therefore, that the difference in task requirements might have affected the subjective location of the click sufficiently to produce the discrepancy noted above.

The purpose of the present experiment was to directly compare the click location method of marking pretyped scripts of the stimulus strings (the Ladefoged and Broadbent method) to the click location method of writing out the stimulus string before marking the perceived location of the click (the Fodor and Bever method) to see if different response requirements result in different click migration patterns for the same set of stimulus sentences. It was predicted that there would be significantly more click migration toward major constituent breaks when the sentence was written out prior to click location than when clicks were simply located on pretyped scripts of the sentences.

\section{METHOD}

\section{Subjects}

Ss were 24 right-handed adults between the ages of 18 and 35 , with hearing levels of $20 \mathrm{~dB}$ ISO or better in both ears at $.5,1$. and $2 \mathrm{KHz}$. All Ss were Caucasians who spoke the general American dialect.

\section{Materials}

Stimulus strings were 24 sentences with specified major constituent breaks (Bever. Lackner. \& Kirk, 1969). A click of approximately $30-\mathrm{msec}$ duration was located at one of three positions within each sentence. The three click locations, as seen in the example below, were (a) two syllables prior to the major constituent break, (b) at the major constituent break, and (c) two syllables after the major constituent break.

\section{(a) (b) (c)}

\section{WHEN HE STOOD UP + MY SONS BOOK FELL FROM THE LOW TABLE}

Three stimulus tapes were prepared, so that each click position would appear one time on each sentence on one of the three stimulus tapes. A strip of foil for triggering a click generator was pasted on the back of the recording tape in one of the three click positions on each sentence, with the foil positioned at each of the three locations on 8 of the 24 stimulus sentences on each tape. The order of click positions was randomized within each stimulus tape.

\section{Stimulus Presentation}

The Ss were assigned randomly to one of two groups of $12 \mathrm{Ss}$ for each click location method. Each $S$ listened to one of the three stimulus tapes while seated by a table in a sound-treated room. Stimulus material was presented biaurally through a matched pair of TDH-39 earphones at $70 \mathrm{~dB}$ ISO. The sentences were presented through one earphone and the clicks through the other earphone. To control for any ear effects (Fodor \& Bever, 1965), one half of the Ss in each group heard the clicks in the right ear and the sentences in the left ear and the other half heard the clicks in the left ear and the sentences in the right ear.

A click of approximately $30 \mathrm{msec}$ was delivered to the earphone from a custom-built click generator produced via an attenuator. The actual click location on each sentence was confirmed by a graphic recording.

The $S_{s}$ in the group required to mark prepared scripts were instructed that they were going to hear a series of sentences on which a click was superimposed. After listening to each sentence with its accompanying superimposed click, the tape recorder would be stopped and the Ss were to turn a page in their booklet and mark the location of the click by placing an arrow over the exact spot (letter or space between words) on the typed copy of the stimulus sentence where the click was perceived as occurring. The $S s$ in the group required to write out the sentence before marking the click location were given similar instructions, except that they were told to turn a page in their booklet and. on the blank page supplied. to write out the stimulus sentence exactly. then mark the location of the perceived click to the nearest let ter or space between the words on their handwritten copy of the stimulus sentence. After completing their respective tasks on each trial, the $S s$ in both groups were instructed to push a button to indicate readiness for the next trial.

\section{Data Analysis}

The click location responses were tabulated for amount and direction of movement. if any. from the objective click location for each individual sentence. Syllables and orthographic spaces between the words were counted as units of measurement. As indicated in the following example. the major constituent boundary served as the zero point. With negative integers indicating syllables and spaces that occurred prior to the major constituent break and positive integers indicating syllables and spaces occurring after the major constituent break:

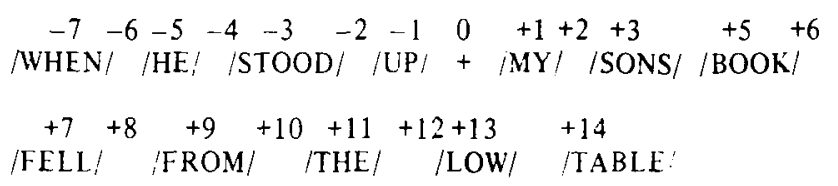

\section{RESULTS}

For the group using the prepared script. $31 \%$ of the responses coincided with the objective click position, as compared to $27 \%$ correct responses for the group required to write out the stimulus sentence before marking the perceived click location. For the combined groups, there were $20 \%$ correct for Click Position a. $43 \%$ correct at Click Location b, and 23\% correct at Click Location c. A 2 by 3 analysis of variance was performed to determine if there were any group or position differences related to correctness of click location without regard to amount or direction of incorrect placement. The difference between groups was not significant, but there was a significant difference between positions $(F=9.55, \quad p<.01 . \quad d f=2 / 44)$, reflecting the greater accuracy of locating the in-break Click Position $b$. The interaction of Groups by Positions was not significant.

For the examination of click migration or movement. only the clicks located two syllables prior to the major constituent break (a) and two syllables after the major constituent break (c) were used. Amount of movement 
was tabulated in terms of the number of syllables the click was displaced toward the break. with click displacement away from the break counted as negative movement. To equate for the unequal number of syllables preceding the first click location and following the last click location on the various sentences. all movements of four or more units were counted as four click location movements. Clicks located in the major constituent break (b) were not used, since they could only constitute movement away from the break. The mean movement toward the break was only 0.44 units $(S D=.31)$ for the script-marking group: whereas the write-out group showed a mean movement toward the break of 0.66 units $(S D=.80)$. There was a positional mean movement toward the break from Click Position $\mathrm{c}$ of 1.4 units $(S D=1.02)$. in contrast to a mean movement of -0.71 units $(S D=1.32)$ away from the break in Click Position a. Positional movements for the script-marking group were -0.94 units $(\mathrm{SD}=1.34$ ) away from the break in Click Position a and +1.02 units $(\mathrm{SD}=99)$ toward the break for Click Position $\therefore$. The respective values for the write-out group were -0.48 units $(\mathrm{SD}=1.61)$ from Position $\mathrm{a}$ and +1.80 units $(\mathrm{SD}=.87)$ from Click Position :. A 2 by 2 analysis of variance demonstrated that there was significantiy more movement toward the major constituent break for the write-out group as compared to the script-marking group $(F=5.60 . p<.05 . d f=1.22)$ and from Position $c$ as compared to Position a $(F=25.95, p<.01 . \mathrm{df}=1 / 22)$. The Group by Position interaction was not significant.

\section{DISCUSSION}

In the group with response requirements corresponding to those used by Fodor and Bever. there was substantial movement toward the major constituent break. whereas the group with response requirements corresponding to those of Ladefoged and Broadbent showed essentially no mean movement from the objective click location. The unequal amounts of movement from the pre- and postbreak click positions revealed a strong left or prior entry bias for both groups in addition to the substantial movement toward the major constituent break for the write-out group. These findings are consistent with previous research in showing different results for the two response methods and consistent with Ladefoged and Broadbent's findings of a prior entry bias.

It seems likely that the different response requirements. as specified by the two different sets of instructions given the $\mathrm{Ss}$ by the two experimental groups. created different perceptual expectations about the listening tasks or differentially affected the storage or retrieval requirements of the listening task. One perceptual or memory expectation was established by asking the Ss to locate the click to the nearest sound or syllable in a word or space between words on prepared scripts. The prepared script seemed to eliminate the necessity for the Ss to process the whole sentence linguistically. All they had to do was listen for a portion of a word or an acoustic space between those words where the click was superimposed, then read the script of the stimulus sentence until the word or words were found and mark the click location accordingly. The task as construed in this manner becomes a matching task. The Ss may or may not linguistically process the stimulus sentence. Even if some linguistic processing does occur (as indicated by the ease of corract location for the in-break click location), it may be of the phonological or morphological rather than the structural tvpe.

The other perceptual or memory set was established by requiring the $S$ not only to listen for the superimposed click but also to listen to the stimulus sentence closely enough that he could write it out exactly before marking the location of the perceived click on his handwritten copy. This task seems more complex than the task above, because Ss had to provide themselves with a handwritten copy of the heard sentence before they were allowed to mark the location of the superimposed click on this copy. If the sentence was not transcribed exactly as spoken, the click location response was voided. The requirement of writing out the stimulus sentence before marking the location of the superimposed click was adopted by Fodor and Bever and the other investigators who used this method in an effort to insure that the Ss were linguistically processing the stimulus sentence. Although we cannot be certain how the listener does process the sentence, the requirement of writing out the sentence does seem to suggest that Ss using this response method must operate on a different level of analysis than Ss using the mark-script method of response. This was borne out by the greater migration of click locations toward the major constituent break by the Ss getting the write-out instructions.

While it had been expected from the results of Ladefoged and Broadbent that the mark-script response method would reveal some prior entry bias. the large proportion of early click locations in both groups was not anticipated. The strong prior entry bias is in accordance with Titchener's law of prior entry. suggesting that Ss do perceive an expected click as occurring prior to its actual location in an unpredictable string of words. Moreover. the effect occurs even in a situation in which the linguistic requirements of the task produce a migration of the click toward the major constituent break.

\section{CONCLUSION}

The results of this experiment clearly demonstrated that different response requirements result in different patterns of click migration. This clarification of the role that response requirements play in studies utilizing modification of the Ladefoged and Broadbent paradigm 
should help to resolve some of the apparent discrepancies in the findings of these studies.

\section{REFERENCES}

Abrams. K., \& Bever, T. G. Syntactic structure modifies attention during speech perception and recognition. Quarterly Journal of Experimental Psychology, 1969, 21, 28-90.

Bever, T. G., Kirk, R., \& Lackner, J. An automatic reflection of syntactic structure. Neuropsychologia, 1969, 7, 23-28.

Bever. T. G., Lackner, J., \& Kirk, R. The underiying structures of sentences are the primary units of immediate speech processing. Perception \& Psychophysics, 1969, 5, 225-234.

Chomsky, N. Aspects of the theory of syntax. Cambridge, Mass: M.I.T. Press, 1965.

Fodor, J. A.. \& Bever, T. G. The psychological reality of linguistic segments. Journal of Verbal Learning \& Verbal Behavior, 1965, 4, 414-420.

Ladefoged, P.. \& Broadbent. D. E. Perception of sequence in auditory events. Quarterly Journal of Experimental Psychology, 1960, 12, 162-170.

Reber, A. S., \& Anderson, J. The perception of clicks in linguistic and nonlinguistic messages. Perception \& Psychophysics, 1970, 8, 81-89.

Scholes, R., Teas, D., Nielsen, D., \& Idzilowski, R. An investigation of sensory and motoric activities in the comprehension of sentences. Quarterly Progress Report, University of Florida Speech Science Laboratory, 1969, 7, 3.

Sedge, R. K. An investigation of displacement of randomized auditory clicks in sentential material. Unpublished paper, Department of Speech. University of Washington, 1968.

Titchener, E. B. Lectures on the experimental psychology of the thought processes. New York: McMillan, 1909.

(Received for publication February 8, 1973; revision received April 27, 1973;

second revision received May 18, 1973.) 